\title{
Distributed Observer Design for Linear Systems under Time-Varying Communication Delay
}

\author{
Kairui Chen $\mathbb{D}^{1,2}$, Junwei Wang $\mathbb{D}^{\circ},{ }^{3}$ Xiaojing Zhong $\mathbb{D}^{1},{ }^{1}$ and Guanyu Lai $\mathbb{D}^{4}$ \\ ${ }^{1}$ School of Mechanical and Electrical Engineering, Guangzhou University, Guangzhou 510006, China \\ ${ }^{2}$ Key Laboratory of System Control and Information Processing, Ministry of Education, Shanghai 200240, China \\ ${ }^{3}$ School of Mathematics and Statistics, Guangdong University of Foreign Studies, Guangzhou 510006, China \\ ${ }^{4}$ School of Automation, Guangdong University of Technology, Guangzhou 510006, China \\ Correspondence should be addressed to Junwei Wang; wangjwlj@gmail.com
}

Received 23 June 2021; Revised 24 July 2021; Accepted 20 August 2021; Published 7 September 2021

Academic Editor: Zhiwei Gao

Copyright (๑) 2021 Kairui Chen et al. This is an open access article distributed under the Creative Commons Attribution License, which permits unrestricted use, distribution, and reproduction in any medium, provided the original work is properly cited.

\begin{abstract}
The paper investigates the state estimation problem of general continuous-time linear systems with the consideration of timevarying communication delay. A solution is proposed in terms of the networked distributed observer, which consists of multiple local observers. Each local observer relies on only part of the system output and exchanges information with neighbors through undirected links modeled by a prespecified communication graph. A simple approach for computing observer parameters is presented by solving a parametric algebraic Riccati equation. Furthermore, by the Lyapunov-Krasovskii stability theorem, an upper bound of the delay could be calculated explicitly and together with the conditions of joint observability and connectivity of the communication graph; the resulting distributed observers work coordinately to achieve an asymptotic estimate of the full plant state. An illustrative example is provided to confirm the analytical results.
\end{abstract}

\section{Introduction}

State estimation or reconstruction is one of the most essential problems in control science and technology. In many control strategies (e.g., state feedback control, process monitoring, and fault detection), we need to have the state variables to construct the controller. In practice, it would be hard to directly obtain all states of an actual system because of technical limitations or perhaps immense cost by using complex sensors [1, 2]. Therefore, there is an urgent necessity to achieve an accurate estimation of the unmeasurable state variables. For deterministic linear time-invariant multivariable systems, the wellknown Luenberger observer [3,4] offers a comprehensive solution to accurately reconstruct the state variables by using the observability of the systems.

During the past decades, based on the Luenberger observer, many observer design schemes were proposed for various linear and nonlinear systems $[5,6]$, switched/hybrid systems [7-9], and systems modeled by partial differential equations or differential equations [10-14]. So far, most works in the literature of the observer design consider the scenario that the observer has access to the full output information of the system to be observed. However, when estimating the states of a spatially deployed system, it is difficult to obtain full information of its output signals individually [15]. This requires us to find a strategy to guarantee an asymptotic estimate of the full plant states while each observer receives only a portion of the measured outputs [16-18]. One feasible strategy to consider this issue is the so-called distributed observer consisting of networked local observers. A key feature of networked distributed observers is that each local observer rely on only its local measured outputs and cooperates with its neighboring observers to estimate the full plant states. This feature can be helpful in formational control of vehicles, autonomous underwater vehicles (AUVs), and spacecrafts [19-21].

Inspired by the extension of Kalman filtering by the idea of distributed control $[22,23]$, the design of the distributed observer for time-invariant linear systems has recently attracted lots of attention. For example, the authors in [24, 25] proposed a network of augmented observers and achieved the right state estimation of discrete-time linear time-invariant systems. 
However, the introduction of an augmented state in each local observer results in the expanded dimensions of the augmented observer, which could induce a heavy computational burden in implementing their proposed schemes. For continuous-time linear time-invariant systems, the networked Luenberger observers proposed in [26-29] provided a feasible solution. The particularity of their formulations is that the effectiveness of the distributed observers can be verified by examining the observability of the system and connectivity of the communication network separately. Along with the idea in [26], different algorithmic procedures to design gain parameters of each local observer using local information of the plant dynamics and its own measurement output were presented in $[30,31]$. However, the distributed observer schemes proposed by the abovementioned works considered ideal interconnection networks without communication time delay among local observers.

In many practical systems, time delays are inevitable, which could degrade the performance of the system or even result instability. Some research studies concerning the time-delay systems have been reported, such as the time-delay state-space system with disturbances [32] and systems with state and input time delays [33]. In network communication systems, the information exchange on the network is often affected by time delay due to physical limitations in communication channels, finite-time information processing, or time-response of actuators [34-37]. As these communication delays may induce instability or poor performance of the systems, they should be taken into consideration to ensure performance of the proposed distributed observer. In [38], an augmented observer, which integrated robust estimation and compensation technique, was proposed for nonlinear systems subjected to unknown measurement delays. Up to now, there are few works on networked distributed observer design for continuous-time linear systems with the consideration communication delays. In $[39,40]$, two kinds of distributed Luenberger observer, tolerating arbitrarily large communication delays were proposed, while their sufficient conditions in both papers required all eigenvalues of the system matrix on the imaginary axis. In addition, the communication delays needed to be constant and their values to be known exactly in $[39,40]$, which is quite restrictive as communication delay is often time varying.

The lack of considering time-varying communication delay and the restriction on the system matrix motivate us to develop a novel distributed observer algorithm for general continuoustime linear systems by assuming the following. (1) The linear plants have general poles, i.e., poles of the system matrix can have zero, negative, or positive real parts. (2) The communication delay in the communication network is time varying. Therefore, the local observers in this paper are deployed to estimate linear plants with general poles under time-varying communication delay. The ability of observing general linear systems is critical for observers as they are generally used in the feedback loops. By solving the parametric algebraic Riccati equation, the gain matrix for local measurement output is determined. Furthermore, by applying the Lyapunov-Krasovskii theorem, we derive the process of computing coupling gains for the information exchange among local observers and the upper bound of the time-varying delay, under which distributed observers achieve omniscience eventually.
The remainder sections of this paper are organized in the following way. Section 2 reviews some necessary preliminaries and introduces the problem statement. In Section 3, we present the detailed design of the distributed observers for linear systems with time-varying communication delay. Simulation results are reported in Section 4. Finally, concluding remarks and further research directions are presented in Section 5.

Let $\mathbb{R}^{+}, \mathbb{R}^{n}$, and $\mathbb{R}^{n \times m}$ stand for the nonnegative real number, $n$-dimensional Euclidean space, and the set of all $n \times$ $m$ real matrices, respectively, $O_{n}$ and $I_{n}$ signify the zero matrix and the identity matrix, $1_{n}$ denotes the $n$-dimensional column vector with all elements as $1, X>0 \quad(X<0)$ means that $X \in \mathbb{R}^{n \times n}$ is a positive definite matrix (negative definite matrix), $\otimes$ is the Kronecker product, and $\operatorname{Diag}\left(\alpha_{1}, \alpha_{2}, \ldots, \alpha_{n}\right)$ stands for a diagonal matrix with diagonal elements $\alpha_{i}$, $i=1,2, \ldots, n$, being real numbers (or a block diagonal matrix with diagonal blocks $\alpha_{i}, i=1,2, \ldots, n$, being matrices). All matrices are assumed to be compatible in this paper.

\section{Preliminaries and Problem Formulation}

2.1. Graph Theory. Let $\mathscr{G}=(\mathscr{V}, \mathscr{E})$ be an undirected graph with the node set $\mathscr{V}=\{1, \ldots, N\}$ and the edge set $\mathscr{E} \subseteq \mathscr{V} \times \mathscr{V}$. An edge in $\mathscr{E}$ is denoted by an unordered pair of distinct nodes $(i, j)$, and $(i, j) \in \mathscr{E}$ if and only if $(j, i) \in \mathscr{E}$. The neighbor set of node $i$ is denoted by $N_{i}=\{j \in \mathscr{V}:(i, j) \in \mathscr{E}\}$. $G=\left[g_{i j}\right] \in \mathbb{R}^{N \times N}$ represents the adjacency matrix associated with $\mathscr{G}$, where $g_{i j}=g_{j i}>0$ if $(i, j) \in \mathscr{E}$ and $g_{i j}=g_{j i}=0$ otherwise. Assuming that there is no self-cycle for each node, i.e., $g_{i i}=0$, correspondingly, the Laplacian matrix $L=\left[l_{i j}\right] \in \mathbb{R}^{N \times N}$ is defined as $l_{i i}=\sum_{k \in N_{i}} g_{i k}$ and $l_{i j}=-g_{i j}$, for $j \neq i$. For an undirected graph $\mathscr{G}$, both its adjacency matrix and Laplacian matrix are symmetric. A sequence of edges $\left(i_{1}, i_{2}\right),\left(i_{2}, i_{3}\right), \ldots,\left(i_{k-1}, i_{k}\right)$ is called a path from node $i_{1}$ to node $i_{k}$. An undirected graph is called connected if, for any node pair $i, j \in \mathscr{V}$, there exists a path from $i$ to $j$.

The following lemma is a well-known result in algebraic graph theory [41].

Lemma 1. For an undirected graph $\mathscr{G}=(\mathscr{V}, \mathscr{E})$ with $N$ nodes, its Laplacian matrix $L$ has $N$ real eigenvalues which can be arranged in an ascending order as $0=\lambda_{1} \leq \lambda_{2} \leq \cdots \leq \lambda_{N}$. Moreover, if $\mathscr{G}$ is connected, $\lambda_{1}=0$ is a simple eigenvalue of $L$ with corresponding eigenvector $1_{N}$.

2.2. Problem Formulation. In this paper, the linear system to be observed is

$$
\begin{aligned}
& \dot{x}=A x, \\
& y=C x=\left[\begin{array}{c}
C_{1} \\
C_{2} \\
\vdots \\
C_{N}
\end{array}\right] x=\left[\begin{array}{c}
y_{1} \\
y_{2} \\
\vdots \\
y_{N}
\end{array}\right],
\end{aligned}
$$

where $x=\left[x_{1}, \ldots, x_{n}\right]^{T} \in \mathbb{R}^{n}$ is the state, $y \in \mathbb{R}^{q}$ is the measurement output, and the matrices $A \in \mathbb{R}^{n \times n}$ and 
$C \in \mathbb{R}^{q \times n}$ are system matrices. We have partitioned the output $y$ as $y=\left[y_{1}^{T}, \ldots, y_{N}^{T}\right]^{T}$ according to the partition of the matrix $C=\left[C_{1}^{T}, \ldots, C_{N}^{T}\right]^{T}$, where $y_{i} \in \mathbb{R}^{q_{i}}$ and $C_{i} \in \mathbb{R}^{q_{i} \times n}$.

For plant (1), with the given undirected communication graph $\mathscr{G}=(\mathscr{V}, \mathscr{E})$, our objective is to construct a group of $N$ networked observers, where each observer computes a state estimate that asymptotically converges to the entire plant state. The following constraints are challenging in the distributed estimation problem: (i) each local observer can only access the portion $y_{i}=C_{i} x$ of the plant output $y=C x$ and (ii) the information exchange among local observers is restricted by a sparsity constraint described by the communication topology, where local observers are represented by nodes and the edges in $\mathscr{E}$ which determine the information links among local observers. In light of the structure of the distributed observers in $[1,39]$, we propose a kind of distributed observer taking the following form:

$$
\begin{aligned}
\dot{\hat{x}}_{i}= & A \widehat{x}_{i}+H_{i}\left(y_{i}-C_{i} \widehat{x}_{i}\right) \\
& +k \sum_{j \in N_{i}} g_{i j}\left(\hat{x}_{j}(t-d(t))\right)-\left(\hat{x}_{i}(t-d(t))\right) .
\end{aligned}
$$

where $\hat{x}_{i}=\left[\hat{x}_{i 1}, \ldots, \hat{x}_{i n}\right]^{T} \in \mathbb{R}^{n}$ is an estimate of the state generated by the $i$ th local observer, $g_{i j}$ shows the relation between observer $i$ and observer $j, N_{i}$ is the set of neighboring observers of observer $i, H_{i} \in \mathbb{R}^{n \times q_{i}}$ is the gain matrix for local output measurements to be determined, and $k>0$ is the coupling strength for neighboring local observers to be designed. $d(t): \mathbb{R}^{+} \longrightarrow \mathbb{R}^{+}$is a time-varying communication delay. The delay is assumed to be bounded by a constant $D>0$ (i.e., $0 \leq d(t) \leq D$ ), which could be estimated by empirical observations or testings. To make the distributed observer (2) work under the delayed communication, we additionally set the initial condition $x(\theta)=\varphi(\theta)$ and $\widehat{x}_{i}(\theta)=\varphi_{i}(\theta)$, for any $\theta \in[-D, 0]$, where $\varphi(\theta)$ and $\varphi_{i}(\theta)$ are known continuous initial state-vector functions.

Remark 1 . In the designed distributed observer (2), the term $\hat{x}_{j}(t-d(t))-\hat{x}_{i}(t-d(t))$ represents the relative state between observer $i$ and observer $j$, which is subject to communication time delay $d(t)$. Therefore, the exactly data of $d(t)$ is not required in the distributed observer. In other words, we intend to measure $\left(\hat{x}_{j}(t)-\hat{x}_{i}(t)\right)$, but the information that we obtain is delayed by the communication network. The time delay $d(t)$ exists inevitably in the communication channel, and we use $d(t)$ to imitate the time delay. Thus, we want to design a kind of distributed observer that considers the delay in order to depress the degradation caused by the delay.

Assumption 1. The undirected graph $\mathscr{G}$ is connected.

Assumption 2. The pair $(A, C)$ is observable.

Remark 2. According to dynamics (2), each local observer recuperates the full plant state $x$ from its local measurements $y_{i}$ and relative delayed information $\hat{x}_{j i}(t-d(t))\left(j \in N_{i}\right)$ of its neighbors. For the special case that there is one $C_{i}$ such that $\left(A, C_{i}\right)$ is detectable, then other observers are unnecessary and the distributed structure becomes trivial. Therefore, we assume the pair $(A, C)$ is detectable but each separated pair $\left(A, C_{i}\right)$ is not; then, exchanging the estimates among neighboring observers is obligatory.

Let $\eta_{i}=\hat{x}_{i}-x$ be the estimation error of the $i$ th observer. Then, the dynamics of observer error $\eta_{i}$ can be obtained from (1) and (2) as follows:

$$
\dot{\eta}_{i}=\left(A-H_{i} C_{i}\right) \eta_{i}-k \sum_{j \in N_{i}} l_{i j} \eta_{j}(t-d(t)),
$$

where $l_{i j}$ is the element of the Laplacian matrix $L$ of $\mathscr{G}$. Stack the local observer errors $\eta_{i}(t)$ to get the joint vector of the observer error $\eta=\left(\eta_{1}^{T}, \eta_{2}^{T}, \ldots, \eta_{N}^{T}\right)^{T}$ and denote $\phi(t)=t-d(t)$. Then, the observer error dynamics governed by (3) can be rewritten as

$$
\begin{aligned}
\dot{\eta}(t) & \\
= & \left(I_{N} \otimes A-\overline{H C}\right) \eta(t)-k\left(L \otimes I_{n}\right) \eta(\phi(t)) \\
= & \left(I_{N} \otimes A-\overline{H C}-k\left(L \otimes I_{n}\right)\right) \eta(t) \\
& +k\left(L \otimes I_{n}\right)(\eta(t)-\eta(\phi(t))) \\
= & \left(I_{N} \otimes A-\overline{H C}-k\left(L \otimes I_{n}\right)\right) \eta(t)+k\left(L \otimes I_{n}\right) \rho(t),
\end{aligned}
$$

where

$$
\begin{aligned}
\rho(t) & =\eta(t)-\eta(\phi(t)), \\
\bar{H} & =\operatorname{Diag}\left\{H_{1}, H_{2}, \ldots, H_{N}\right\}, \\
\bar{C} & =\operatorname{Diag}\left\{C_{1}, C_{2}, \ldots, C_{N}\right\} .
\end{aligned}
$$

Before proceeding further, we first introduce the concept of asymptotic omniscience as stated in [25].

Definition 1. For plant (1), the distributed observer (2) is said to achieve asymptotic omniscience if $\lim _{t \longrightarrow+\infty}\left\|\eta_{i}(t)\right\|=\lim _{t \longrightarrow+\infty}\left\|\widehat{x}_{i}(t)-x(t)\right\|=0$, i.e., each observer state $\hat{x}_{i}(t)$ converges to $x(t)$ asymptotically.

Remark 3. From the multiagent point of view, each local observer in (2) is in the situation of a follower and system (1) to be observed is analogous to the leader. Thus, the dynamical structure of (1) and (2) is in fact a special case of the leader-following multiagent system. A difference lies in that each follower often receives full state vector of the leader, while each local observer in (2) has access to only partial information of the output [39]. To the best of the authors' knowledge, rare result on the consensus problem of such a type of multiagent systems was reported by researchers. Therefore, the problem of designing an observer is still an open problem for the general form (2), especially with the consideration of time-varying communication delay.

In what follows, we will focus on computing the coupling strength $k$ and the observer gain $H_{i}$ such that the designed distributed observer (2) with time-varying communication delay $d(t)$ achieves omniscience asymptotically. 


\section{Distributed Observer for General Linear Systems}

In this section, we are going to design distributed observers for general linear system (1), where the matrix $A$ is general, i.e., may have eigenvalues with zero, negative, or positive real parts. Two kinds of distributed observer algorithms, with communication delay and without communication delay, are designed with rigorous proofs.

Before providing the distributed observer design method, some key technical lemmas for the design and proof of the distributed observer are introduced.

Lemma 2 (see [42]). For $(A, C)$ satisfying Assumption 2, let

$$
\epsilon>-2 \min \{\operatorname{Re}(\lambda(A))\},
$$

where $\operatorname{Re}(\lambda(A))$ is the real parts of $\lambda(A)$ which are eigenvalues of $A$. Then, a unique positive definite solution of the following ARE,

$$
P A^{T}+A P-P C^{T} C P=-\epsilon P,
$$

is given by $P(\epsilon)=W^{-1}(\epsilon)$, where $W(\epsilon)$ can be obtained by solving the following Lyapunov equation:

$$
W\left(A+\frac{\epsilon}{2} I_{n}\right)+\left(A+\frac{\epsilon}{2} I_{n}\right)^{T} W=C^{T} C .
$$

Moreover, $(\mathrm{d} P(\epsilon) / d(\epsilon))>0$ and $P(\epsilon)$ has the following properties:

(i) $\operatorname{tr}\left(C P(\epsilon) C^{T}\right)=2 \operatorname{tr}(A)+n \epsilon$

(ii) $P(\epsilon) C^{T} C P(\epsilon) \leq(2 \operatorname{tr}(A)+n \epsilon) P(\epsilon)$

Lemma 3. Let $(A, C)$ satisfy Assumption 2 and $P(\epsilon)>0$ be the solution to (7). Then, we have

$$
A^{T} P^{-1} A \leq \zeta(\epsilon) P^{-1},
$$

where

$$
\begin{aligned}
\zeta(\epsilon)= & \left.\frac{1}{2}(2 \operatorname{tr}(A)+n \epsilon)^{2}-\frac{1}{2} \epsilon(2 \operatorname{tr}(A)+n \epsilon)\right) \\
& -\epsilon \operatorname{tr}(A)-\operatorname{tr}\left(A^{2}\right) .
\end{aligned}
$$

Proof. From the fact $\operatorname{tr}(X Y)=\operatorname{tr}(Y X)$, we have

$$
\begin{aligned}
A^{T} P^{-1} A & =P^{-1} P A^{T} P^{-1} A P P^{-1} \\
& =P^{-(1 / 2)}\left(P^{-(1 / 2)} P A^{T} P^{-1} A P P^{-(1 / 2)}\right) P^{-(1 / 2)} \\
& \leq \operatorname{tr}\left(P^{-(1 / 2)} P A^{T} P^{-1} A P P^{-(1 / 2)}\right) P^{-1} \\
& =\operatorname{tr}\left(P A^{T} P^{-1} A\right) P^{-1} .
\end{aligned}
$$

Postmultiplying the two sides of ARE (7) with $P^{-1} A$ gives

$$
P A^{T} P^{-1} A+A^{2}-P C^{T} C A=-\epsilon A,
$$

which leads to

$$
\operatorname{tr}\left(P A^{T} P^{-1} A\right)=\operatorname{tr}\left(P C^{T} C A\right)-\varepsilon \operatorname{tr}(A)-\operatorname{tr}\left(A^{2}\right) .
$$

Using ARE (7) again, we obtain

$$
\begin{aligned}
\operatorname{tr} & \left(P C^{T} C A\right) \\
& =\operatorname{tr}\left(C^{T} C A P\right)=\operatorname{tr}\left(C^{T} C\left(P C^{T} C P-P A^{T}-\epsilon P\right)\right) \\
& =\operatorname{tr}\left(\left(C P C^{T}\right)^{2}\right)-\operatorname{tr}\left(P C^{T} C A\right)-\epsilon \operatorname{tr}\left(C P C^{T}\right) .
\end{aligned}
$$

Notice that $\operatorname{tr}(X Y) \leq \operatorname{tr}(X) \operatorname{tr}(Y)$ for $X \geq 0$ and $Y \geq 0$. Then, it follows from (14) and Lemma 2 that

$$
\begin{aligned}
\operatorname{tr}\left(P C^{T} C A\right) & =\frac{1}{2} \operatorname{tr}\left(\left(C P C^{T}\right)^{2}\right)-\frac{1}{2} \epsilon \operatorname{tr}\left(C P C^{T}\right) \\
& \leq \frac{1}{2}\left(\operatorname{tr}\left(C P C^{T}\right)\right)^{2}-\frac{1}{2} \epsilon \operatorname{tr}\left(C P C^{T}\right) \\
& \left.=\frac{1}{2}(2 \operatorname{tr}(A)+n \epsilon)^{2}-\frac{1}{2} \epsilon(2 \operatorname{tr}(A)+n \epsilon)\right) .
\end{aligned}
$$

Substituting (15) into (13) gives

$$
\begin{aligned}
\operatorname{tr}\left(P A^{T} P^{-1} A\right)= & \operatorname{tr}\left(P C^{T} C A\right)-\epsilon \operatorname{tr}(A)-\operatorname{tr}\left(A^{2}\right) \\
\leq & \left.\frac{1}{2}(2 \operatorname{tr}(A)+n \epsilon)^{2}-\frac{1}{2} \epsilon(2 \operatorname{tr}(A)+n \epsilon)\right) \\
& -\epsilon \operatorname{tr}(A)-\operatorname{tr}\left(A^{2}\right) .
\end{aligned}
$$

Consequently, inequality (9) follows from (11) and (16). This completes the proof.

Lemma 4 (Schur complement [43]). For a given symmetric matrix $S=\left[\begin{array}{ll}S_{11} & S_{12} \\ S_{12}^{T} & S_{22}\end{array}\right]$ with $S_{11}=S_{11}^{T}$ and $S_{22}=S_{22}^{T}, S<0$ is equivalent to $S_{11}<0, S_{22}-S_{12}^{T} S_{11}^{-1} S_{12}<0$.

Lemma 5 (Jensen's inequality [44]). Let $t_{1}$ and $t_{2}$ be scalars with $t_{2} \geq t_{1}$, and a vector-valued function $\eta(t):\left[t_{1}\right.$, $\left.t_{2}\right] \longrightarrow \mathbb{R}^{n}$ so that the related integrals are well defined. Then, for any positive definite matrix $P \in \mathbb{R}^{n \times n}$, we have the following inequality:

$$
\left(\int_{t_{1}}^{t_{2}} \eta(t) \mathrm{d} t\right)^{T} P\left(\int_{t_{1}}^{t_{2}} \eta(t) \mathrm{d} t\right) \leq\left(t_{2}-t_{1}\right) \int_{t_{1}}^{t_{2}} \eta^{T}(t) P \eta(t) \mathrm{d} t .
$$

Based on the above preparations, we are ready to propose the following theorem.

Theorem 1. Consider the linear system (1) under Assumptions 1 and 2. Let $0=\lambda_{1}<\lambda_{2} \leq \cdots \leq \lambda_{N}$ denote $N$ real eigenvalues of $L$ and design feedback gain $H_{i}$ and coupling strength $k$ in the distributed observer (2) as

$$
H_{i}=\frac{N}{2} P(\epsilon) C_{i}^{T}, k>\frac{\rho_{1}(\epsilon)}{\lambda_{2}},
$$

where $P(\epsilon)>0$ is the solution to (7) and 


$$
\begin{aligned}
\rho_{1}(\epsilon)= & (4 N-3)(2 \operatorname{tr}(A)+n \epsilon)+1.5 \epsilon \\
& +\frac{2}{\epsilon}(1+N)^{2}(2 \operatorname{tr}(A)+n \epsilon)^{2} .
\end{aligned}
$$

Then, the distributed observer (2) achieves asymptotic omniscience if the upper bound $D$ of the time-varying delay $d(t)$ satisfies

$$
D<\sqrt{\frac{\epsilon}{3 \epsilon k^{2} \lambda_{N}^{2}+2 k \lambda_{N} \rho_{2}(\epsilon)}},
$$

where

$$
\begin{aligned}
\rho_{2}(\epsilon)= & \left(3+1.5 N^{2}\right)(2 \operatorname{tr}(A)+n \epsilon)^{2}+3 k^{2} \lambda_{N}^{2} \\
& -3 \epsilon(2 \operatorname{tr}(A)+n \epsilon)-6 \epsilon \operatorname{tr}(A)-6 \operatorname{tr}\left(A^{2}\right) .
\end{aligned}
$$

Proof. It can be seen that the distributed observer (2) achieves asymptotic omniscience if and only if the observer error system (4) is asymptotically stable. Thus, we choose the following Lyapunov functional candidate to prove the convergence of the global observer error system (4):

$$
V(t)=V_{1}(t)+V_{2}(t)
$$

where

$$
\begin{aligned}
& V_{1}(t)=\eta^{T}(t) \bar{P} \eta(t), \\
& V_{2}(t)=\sigma \int_{-D}^{0} \int_{t+\theta}^{t} \dot{\eta}^{T}(s) \bar{P} \dot{\eta}(s) \mathrm{d} s \mathrm{~d} \theta,
\end{aligned}
$$

and $\bar{P}=I_{N} \otimes P^{-1}$, and $\sigma>0$ is a scalar to be designed.

Along the solutions of (4) with $H_{i}$ in (19), computing the derivative of $V_{1}(t)$ yields

$$
\begin{aligned}
\dot{V}_{1}(t) & \\
= & \eta^{T}(t)\left(I_{N} \otimes\left(A^{T} P^{-1}+P^{-1} A\right)\right) \eta(t)-N \eta^{T}(t) \bar{C}^{T} \bar{C} \eta(t) \\
& -2 k \eta^{T}(t)\left(L \otimes P^{-1}\right) \eta(t)+2 k \eta^{T}(t)\left(L \otimes P^{-1}\right) \rho(t) \\
\leq & \eta^{T}(t)\left(I_{N} \otimes\left(A^{T} P^{-1}+P^{-1} A\right)\right) \eta(t)-N \eta^{T}(t) \bar{C}^{T} \bar{C} \eta(t) \\
& -k \eta^{T}(t)\left(L \otimes P^{-1}\right) \eta(t)+k \rho^{T}(t)\left(L \otimes P^{-1}\right) \rho(t) \\
= & \eta^{T}(t) \Pi(k) \eta(t)+k \rho^{T}(t)\left(L \otimes P^{-1}\right) \rho(t),
\end{aligned}
$$

where

$$
\begin{aligned}
\Pi(k) & =I_{N} \otimes\left(A^{T} P^{-1}+P^{-1} A\right)-N \bar{C}^{T} \bar{C}-k L \otimes P^{-1} \\
& =\operatorname{Diag}\left\{\Upsilon_{1}, \Upsilon_{2}, \ldots, \Upsilon_{N}\right\}-k L \otimes P^{-1},
\end{aligned}
$$

with $\Upsilon_{i}=A^{T} P^{-1}+P^{-1} A-N C_{i}^{T} C_{i}$. Then, according to (7), we have $\sum_{i=1}^{N} \Upsilon_{i}=N\left(A^{T} P^{-1}+P^{-1} A-\sum_{i=1}^{N} C_{i}^{T} C_{i}\right)=-\epsilon N P^{-1}$.

To analyze $\Pi(k)$, we first show that, for any scalar $\epsilon>0$, there is a $k>0$ such that

$$
\Pi(k)<-\frac{1}{2} \epsilon \bar{P} .
$$

By Assumption 1 and Lemma 1, we know that $(1 / \sqrt{N}) 1_{N}$ is the right eigenvector of $L$ associated with the zero eigenvalue. Let $U=\left[(1 / \sqrt{N}) 1_{N}, U_{1}\right]$ be the orthogonal matrix such that

$$
U^{T} L U=\operatorname{Diag}\{0, \widehat{L}\}, U^{-1}=U^{T},
$$

where $\widehat{L}=\operatorname{Diag}\left\{\lambda_{2}, \ldots, \lambda_{N}\right\}$. By premultiplying both sides of (26) with $U^{T} \otimes I_{n}$ and postmultiplying with $U \otimes I_{n}$, we have

$$
\left(U^{T} \otimes I_{n}\right) \Pi(k)\left(U \otimes I_{n}\right)<-\frac{1}{2} \epsilon \bar{P},
$$

which can be rewritten as in (29)

$$
\left(\begin{array}{cc}
-\frac{1}{2} \epsilon P^{-1} & \frac{1}{\sqrt{N}}\left(1_{N}^{T} \otimes I_{n}\right) \bar{Y}\left(U_{1} \otimes I_{n}\right) \\
* & \left(U_{1}^{T} \otimes I_{n}\right) \bar{Y}\left(U_{1} \otimes I_{n}\right)+\left(\frac{1}{2} \epsilon I_{N-1}-k \widehat{L}\right) \otimes P^{-1}
\end{array}\right)<0 .
$$

By the Schur complement in Lemma 4, (29) is equivalent to

$$
\begin{aligned}
& \left(U_{1}^{T} \otimes I_{n}\right) \bar{Y}\left(U_{1} \otimes I_{n}\right)+\left(\frac{1}{2} \epsilon I_{N-1}-k \widehat{L}\right) \otimes P^{-1} \\
& +\frac{2}{\epsilon N}\left(U_{1}^{T} \otimes I_{n}\right) \bar{Y}\left(1_{N} 1_{N}^{T} \otimes P\right) \bar{Y}\left(U_{1} \otimes I_{n}\right)<0,
\end{aligned}
$$

where $\bar{Y}=\operatorname{Diag}\left\{\Upsilon_{1}, \Upsilon_{2}, \ldots, \Upsilon_{N}\right\}$.

From Lemma 2, we can get $\operatorname{tr}\left(C_{i} P C_{i}^{T}\right) \leq \operatorname{tr}\left(C P C^{T}\right)=$ $2 \operatorname{tr}(A)+n \epsilon$ and $C_{i}^{T} C_{i} \leq C^{T} C \leq(2 \operatorname{tr}(A)+n \epsilon) P^{-1}$, and then, $C_{i}^{T} C_{i} P C_{i}^{T} C_{i} \leq C_{i}^{T} \quad \operatorname{tr}\left(C_{i} P C_{i}^{T}\right) C_{i} \leq(2 \operatorname{tr}(A)+n \epsilon) C_{i}^{T} C_{i} \leq(2 \operatorname{tr}$ $(A)+n \epsilon)^{2} P^{-1}$. Hence, it can be verified that

$$
\begin{aligned}
\Upsilon_{i}= & -\epsilon P^{-1}+C^{T} C-N C_{i}^{T} C_{i} \\
\leq & -\epsilon P^{-1}+C^{T} C \leq(2 \operatorname{tr}(A)+n \epsilon-\epsilon) P^{-1} \\
\Upsilon_{i} P \Upsilon_{i}= & \epsilon^{2} P^{-1}-2 \epsilon C^{T} C+2 \epsilon N C_{i}^{T} C_{i}+C^{T} C P C^{T} C \\
& -N C^{T} C P C_{i}^{T} C_{i}-N C_{i}^{T} C_{i} P C^{T} C+N^{2} C_{i}^{T} C_{i} P C_{i}^{T} C_{i} \\
\leq & \epsilon^{2} P^{-1}+2 \epsilon(N-1) C^{T} C+(N+1) C^{T} C P C^{T} C \\
& +\left(N^{2}+N\right) C_{i}^{T} C_{i} P C_{i}^{T} C_{i} \\
\leq & {\left[\epsilon^{2}+2 \epsilon(N-1)(2 \operatorname{tr}(A)+n \epsilon)\right.} \\
& \left.+(1+N)^{2}(2 \operatorname{tr}(A)+n \epsilon)^{2}\right] P^{-1},
\end{aligned}
$$

which implies that

$$
\left(U_{1}^{T} \otimes I_{n}\right) \bar{\Upsilon}\left(U_{1} \otimes I_{n}\right) \leq(2 \operatorname{tr}(A)+n \epsilon-\epsilon)\left(I_{N-1} \otimes P^{-1}\right),
$$

and 


$$
\begin{aligned}
&\left(U_{1}^{T} \otimes I_{n}\right) \bar{\Upsilon}\left(1_{N} 1_{N}^{T} \otimes P\right) \bar{Y}\left(U_{1} \otimes I_{n}\right) \\
& \leq N\left(U_{1}^{T} \otimes I_{n}\right) \bar{\Upsilon}\left(I_{N} \otimes P\right) \bar{\Upsilon}\left(U_{1} \otimes I_{n}\right) \\
& \leq N\left[\epsilon^{2}+2 \epsilon(N-1)(2 \operatorname{tr}(A)+n \epsilon)\right. \\
&\left.+(1+N)^{2}(2 \operatorname{tr}(A)+n \epsilon)^{2}\right]\left(I_{N-1} \otimes P^{-1}\right),
\end{aligned}
$$

where $U_{1}^{T} U_{1}=I_{N-1}$ has been used.

Combining (32) and (33), the left-hand side of inequality (27) satisfies

$$
\begin{aligned}
\left(U_{1}^{T} \otimes I_{n}\right) \bar{\Upsilon}\left(U_{1} \otimes I_{n}\right) & +\left(\frac{1}{2} \epsilon I_{N-1}-k \widehat{L}\right) \otimes P^{-1} \\
& +\frac{2}{\epsilon N}\left(U_{1}^{T} \otimes I_{n}\right) \bar{\Upsilon}\left(1_{N} 1_{N}^{T} \otimes P\right) \bar{\Upsilon}\left(U_{1} \otimes I_{n}\right) \\
& \leq-\left(k \lambda_{2}-\rho_{1}(\epsilon)\right)\left(I_{N-1} \otimes P^{-1}\right),
\end{aligned}
$$

with $\rho_{1}(\epsilon)$ as defined in (19). By choosing the coupling strength $k$ as in (18), the inequality (26) is thus proved.

We now simplify the term $k \rho^{T}(t)\left(L \otimes P^{-1}\right) \rho(t)$ in (24). By Jensen's inequality in Lemma 5 , one obtains

$$
\begin{aligned}
& k \rho^{T}(t)\left(L \otimes P^{-1}\right) \rho(t) \\
& =k\left(\int_{\phi(t)}^{t} \dot{\eta}(s) \mathrm{d} s\right)^{T}\left(L \otimes P^{-1}\right)\left(\int_{\phi(t)}^{t} \dot{\eta}(s) \mathrm{d} s\right) \\
& \leq k(t-\phi(t)) \int_{\phi(t)}^{t} \dot{\eta}^{T}(s)\left(L \otimes P^{-1}\right) \dot{\eta}(s) \mathrm{d} s \\
& \leq k \lambda_{N} D \int_{t-D}^{t} \dot{\eta}^{T}(s) \bar{P} \dot{\eta}(s) \mathrm{d} s,
\end{aligned}
$$

where we use the knowledge that $d(t)$ is bounded.

Substituting inequalities (26) and (35) into (24) gives

$$
\dot{V}_{1}(t) \leq-\frac{1}{2} \epsilon \eta^{T}(t) \bar{P} \eta(t)+k \lambda_{N} D \int_{t-D}^{t} \dot{\eta}^{T}(s) \bar{P} \dot{\eta}(s) \mathrm{d} s .
$$

Next, the derivative of $V_{2}(t)$ along (4) with $H_{i}$ in (18) is

$$
\begin{aligned}
\dot{V}_{2}(t) & =\sigma \int_{-D}^{0}\left(\dot{\eta}^{T}(t) \bar{P} \dot{\eta}(t)-\dot{\eta}^{T}(t+\theta) \bar{P} \dot{\eta}(t+\theta)\right) \mathrm{d} \theta \\
& =\sigma D \dot{\eta}^{T}(t) \bar{P} \dot{\eta}(t)-\sigma \int_{t-D}^{t} \dot{\eta}^{T}(s) \bar{P} \dot{\eta}(s) \mathrm{d} s .
\end{aligned}
$$

By Lemma 3 and Young's inequality, the first term in (37) becomes

$$
\begin{aligned}
& \sigma D \dot{\eta}^{T}(t) \bar{P} \dot{\eta}(t) \\
& =\sigma D \eta^{T}\left(I_{N} \otimes A-\overline{H C}\right)^{T} \bar{P}\left(I_{N} \otimes A-\overline{H C}\right) \eta-2 \sigma D k \eta^{T} \\
& \left(L^{T} \otimes P^{-1}\right)\left(I_{N} \otimes A-\overline{H C}\right) \eta+\sigma D k^{2} \eta^{T}\left(L^{T} L \otimes P^{-1}\right) \eta \\
& \quad+2 \sigma D k \eta^{T}\left(I_{N} \otimes A-\overline{H C}\right)^{T}\left(L \otimes P^{-1}\right) \rho \\
& \quad-2 \sigma D k^{2} \eta^{T}\left(L^{T} L \otimes P^{-1}\right) \rho+\sigma D k^{2} \rho^{T}\left(L^{T} L \otimes P^{-1}\right) \rho
\end{aligned}
$$

$$
\begin{gathered}
\quad \leq 3 \sigma D \eta^{T}(t)\left(I_{N} \otimes A-\overline{H C}\right)^{T} \bar{P} \\
\left(I_{N} \otimes A-\overline{H C}\right) \eta(t)+3 \sigma D k^{2} \lambda_{N}^{2} \eta^{T}(t) \bar{P} \eta(t) \\
\quad+3 \sigma k^{2} \lambda_{N}^{2} D^{2} \int_{t-D}^{t} \dot{\eta}^{T}(s) \bar{P} \dot{\eta}(s) \mathrm{d} s \\
=3 \sigma D \eta^{T} \operatorname{Diag}\left\{\Psi_{1}, \Psi_{2}, \ldots, \Psi_{N}\right\} \eta(t)+3 \sigma D k^{2} \lambda_{N}^{2} \eta^{T}(t) \\
\bar{P} \eta(t)+3 \sigma k^{2} \lambda_{N}^{2} D^{2} \int_{t-D}^{t} \dot{\eta}^{T}(s) \bar{P} \dot{\eta}(s) \mathrm{d} s
\end{gathered}
$$

where

$$
\begin{aligned}
\Psi_{i}= & A^{T} P^{-1} A-\frac{N}{2} A^{T} C_{i}^{T} C_{i}-\frac{N}{2} C_{i}^{T} C_{i} A \\
& +\frac{N^{2}}{4} C_{i}^{T} C_{i} P C_{i}^{T} C_{i} \\
\leq & 2 A^{T} P^{-1} A+\frac{N^{2}}{2} C_{i}^{T} C_{i} P C_{i}^{T} C_{i} \\
\leq & 2 \zeta(\epsilon) P^{-1}+\frac{N^{2}}{2}(2 \operatorname{tr}(A)+n \epsilon)^{2} P^{-1},
\end{aligned}
$$

with $\zeta(\epsilon)$ as defined in (10). With $\rho_{2}(\epsilon)$ in (21), we obtain

$$
\begin{aligned}
& \sigma D \dot{\eta}^{T}(t) \bar{P} \dot{\eta}(t) \\
& \quad \leq \sigma D\left(6 \zeta(\epsilon)+\frac{3}{2} N^{2}(2 \operatorname{tr}(A)+n \epsilon)^{2}+3 k^{2} \lambda_{N}^{2}\right) \eta^{T}(t) \\
& \bar{P} \eta(t)+3 \sigma k^{2} \lambda_{N}^{2} D^{2} \int_{t-D}^{t} \dot{\eta}^{T}(s) \bar{P} \dot{\eta}(s) \mathrm{d} s \\
& \quad \leq \sigma D \rho_{2}(\epsilon) \eta^{T}(t) \bar{P} \eta(t)+3 \sigma k^{2} \lambda_{N}^{2} D^{2} \int_{t-D}^{t} \dot{\eta}^{T}(s) \bar{P} \dot{\eta}(s) \mathrm{d} s .
\end{aligned}
$$

Then, substitution of (36) and (37) with (40) into the derivative of $V(t)$ in (22) results in

$$
\begin{aligned}
\dot{V}(t)= & \dot{V}_{1}(t)+\dot{V}_{2}(t) \\
\leq & -\frac{1}{2} \epsilon \eta^{T}(t) \bar{P} \eta(t)+k \lambda_{N} D \int_{t-D}^{t} \dot{\eta}^{T}(s) \bar{P} \dot{\eta}(s) \mathrm{d} s \\
& +\sigma D \dot{\eta}^{T}(t) \bar{P} \dot{\eta}(t)-\sigma \int_{t-D}^{t} \dot{\eta}^{T}(s) \bar{P} \dot{\eta}(s) \mathrm{d} s \\
\leq & -\left(\frac{1}{2} \epsilon-\sigma D \rho_{2}(\epsilon)\right) \eta^{T}(t) \bar{P} \eta(t) \\
& +\left(3 \sigma k^{2} \lambda_{N}^{2} D^{2}+k \lambda_{N} D-\sigma\right) \int_{t-D}^{t} \dot{\eta}^{T}(s) \bar{P} \dot{\eta}(s) \mathrm{d} s .
\end{aligned}
$$

Therefore, by taking

$$
\sigma=\frac{k \lambda_{N} D}{1-3 k^{2} \lambda_{N}^{2} D^{2}}
$$


which is positive based on the delay bound condition (20), we have

$$
\begin{aligned}
\dot{V}(t) & \leq-\left(\frac{1}{2} \epsilon-\sigma D \rho_{2}(\epsilon)\right) \eta^{T}(t) \bar{P} \eta(t) \\
& \leq-\left(\frac{1}{2} \epsilon-\frac{k \lambda_{N} D^{2} \rho_{2}(\epsilon)}{1-3 k^{2} \lambda_{N}^{2} D^{2}}\right) \eta^{T}(t) \bar{P} \eta(t) .
\end{aligned}
$$

Note that the delay bound condition (20) implies $1 / 2 \epsilon>k \lambda_{N} D^{2} \rho_{2}(\epsilon) / 1-3 k^{2} \lambda_{N}^{2} D^{2}$, and thus, $\dot{V}(t)<0$. By the Lyapunov-Krasovskii stability theorem [45], asymptotic omniscience of the distributed observer (2) has been achieved under the gain parameter choice satisfying (18)-(21).

Remark 4. According to the sufficient condition (18)-(21) for the distributed observer in Theorem 1, the parameter $\epsilon$ needs to be chosen before we can construct the observers. Firstly, $\epsilon$ is a parameter in ARE (7), and $(d / \mathrm{d} \epsilon) P(\epsilon)>0$. Therefore, $\epsilon$ is usually a small parameter to keep $H_{i}$ as a small feedback gain. Secondly, there is a low bound for $\epsilon$ according to (6) in Lemma 2. Moreover, note that $\epsilon$ affects the upper bound of the time-varying delay $d(t)$ according to (20) and affects the convergence rate of the distributed observers according to (43). These tradeoffs need to be considered when selecting a proper $\epsilon$.

Remark 5. A similar setting of state estimation problem was discussed in [39], where the time delay was assumed to be constant and known. However, the above proof demonstrates that the stability analysis of the observer error system (4) involves only the information on the bound $D$ of the time-varying delay $d(t)$, without requiring its exact knowledge. Therefore, our proposed distributed observer scheme is applicable to more general cases (e.g., fast-varying communication delay) and is also robust to the uncertainty in the communication time delay.

For the special case with no communication time delays, the distributed observer (2) becomes

$$
\dot{\hat{x}}_{i}=A \widehat{x}_{i}+H_{i}\left(y_{i}-C_{i} \hat{x}_{i}\right)+k \sum_{j \in N_{i}} g_{i j}\left(\hat{x}_{j}(t)-\hat{x}_{i}(t)\right) .
$$

Regarding this circumstance, a sufficient condition for the distributed observer (44) to achieve asymptotic omniscience is described by the following corollary.

Corollary 1. Consider the linear system (1) under Assumptions 1 and 2. Then, the distributed observer (44) achieves asymptotic omniscience with the feedback gain $H_{i}=$ $(N / 2) P(\epsilon) C_{i}^{T}$ if

$$
k>\frac{\rho_{1}(\epsilon)}{2 \lambda_{2}},
$$

where $P(\epsilon)$ is the solution to (7), $\lambda_{2}>0$ is the second smallest eigenvalue of $L$, and $\rho_{1}(\epsilon)$ is as in (19).
Proof. Without the communication delay, the error system between (1) and (44) reduces to

$$
\dot{\eta}(t)=\left(I_{N} \otimes A-\overline{H C}-k\left(L \otimes I_{n}\right)\right) \eta(t),
$$

where $\bar{H}$ and $\bar{C}$ are as defined in (4). To prove the omniscience of this distributed observer, a Lyapunov function for (46) can be defined as follows:

$$
V(t)=\eta^{T}(t) \bar{P} \eta(t),
$$

where $\bar{P}=I_{N} \otimes P^{-1}$.

For the feedback gain, $H_{i}=(N / 2) P(\epsilon) C_{i}^{T}$, and the coupling strength $k$ in (45), the fact that the error system (46) is asymptotically stable is shown similar with the corresponding proof in Theorem 1 .

Remark 6. Both observers (2) and (44) can be implemented in a distributed manner, since they compute the state estimates of the local observer by the information exchanged with neighbors. However, the choice of the coupling gain $k$ in (19) and (45) needs some global information (e.g., $\lambda_{2}$ ) of the communication graph $\mathscr{G}$, whose design is not a decentralized one. Without the global knowledge of the communication graph, one can take $k$ sufficiently large to make inequality (19) or (45) hold. Another feasible method to select the coupling strength $k$ in (2) and (44) is the adaptive regulation law [46, 47].

\section{Numerical Example}

Consider the following system matrices for system (1):

$$
\begin{aligned}
A & =\left[\begin{array}{cccc}
-0.04 & 0 & 2 & 0 \\
0 & 0.02 & 0 & -1 \\
0 & 0 & 0 & -1 \\
0 & 0 & 1 & 0
\end{array}\right], \\
C & =\left[\begin{array}{l}
C_{1} \\
C_{2} \\
C_{3}
\end{array}\right],
\end{aligned}
$$

where $C_{1}=[2,0,0,0], C_{2}=[0,2,0,0]$, and $C_{3}=[0,0,0,2]$. The open-loop dynamics of this system is exponentially unstable, since system matrix $A$ has eigenvalues $\sigma(A)=\{-0.04,0.02, \pm i\}$.

By direct calculation, we have $(A, C)$ is detectable, $\operatorname{tr}(A)=-0.02$, and $\operatorname{tr}\left(A^{2}\right)=-2.0$. It can be easily verified that none of $\left(A, C_{i}\right)$ is detectable. Hence, we construct a network of $N=3$ local observers, and observer $i$ can obtain $y_{i}=C_{i} x$ instead of $y=C x$. The relationship among these observers is given in Figure 1. For simplicity, here, we suppose that all the weights are set as 2 . The corresponding Laplacian matrix is given by

$$
L=2\left[\begin{array}{ccc}
1 & -1 & 0 \\
-1 & 2 & -1 \\
0 & -1 & 1
\end{array}\right] \text {, }
$$

whose eigenvalues are $\lambda_{1}(L)=0, \lambda_{2}(L)=2$, and $\lambda_{3}(L)=6$. 


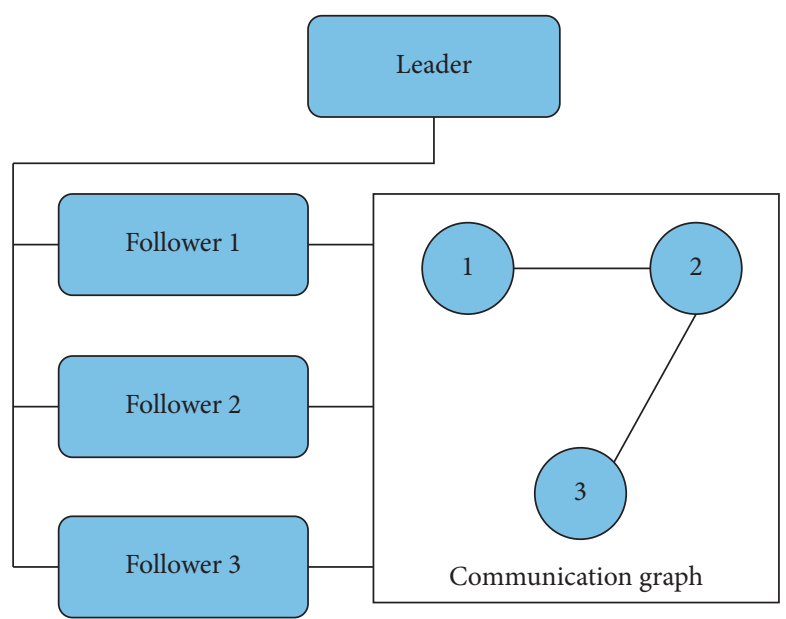

FIGURE 1: Framework of the networked distributed observer consisting of $N=3$ local observers.
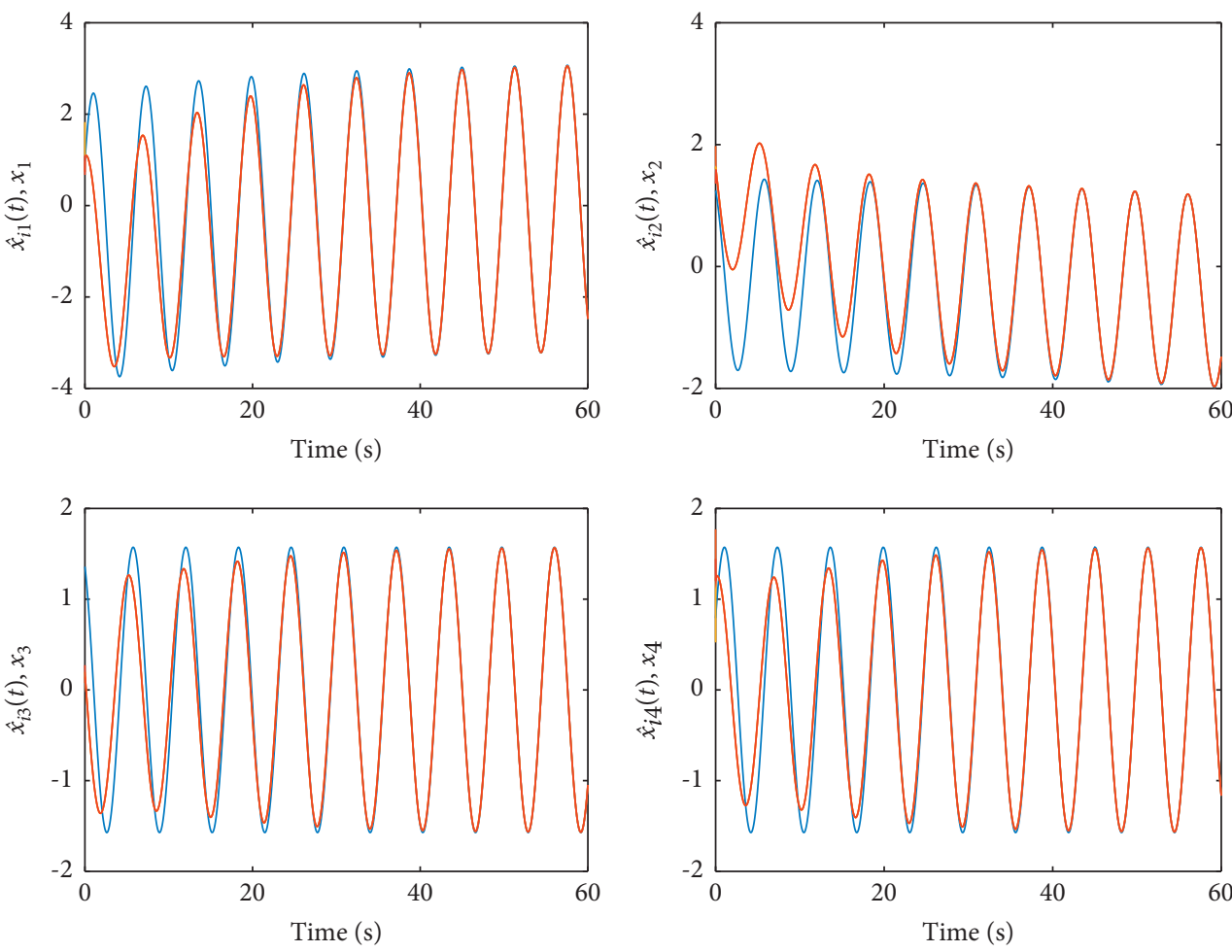

FIgURE 2: Observer states $\widehat{x}_{i}(t)(i=1,2,3)$ and the plant state $x(t)$ for time-varying communication delay.

By selecting the parameter $\epsilon=0.18$, the solution of ARE (7) is calculated as

$$
P=\left[\begin{array}{cccc}
0.0852 & 0.0001 & 0.0060 & 0.0299 \\
0.0001 & 0.0696 & 0.0148 & -0.0020 \\
0.0060 & 0.0148 & 0.0156 & 0.0009 \\
0.0299 & -0.0020 & 0.0009 & 0.0151
\end{array}\right] .
$$

In the delayed case, by calculating (18) and (19), we have the coupling strength $k>44.40$. Let $k=44.5$, the corresponding delay bound can be calculated by (20). Figures 2 and
3 show the trajectories and the observer errors of the distributed observers with a time-varying communication delay $d(t)=0.1 \sin (t)^{2}$. According to the results, we can find that all observers follow the states of the linear plant with very small errors. For the delay-free case, by (41), we have $k>22.2$, and other parameters are the same with the delayed case. With $k=22.3$, Figures 4 and 5 show the trajectories and the observer errors of the distributed observers without communication delay. The delay-free case allows a smaller coupling strength with satisfied estimation results. The results of the simulations verify the effectiveness of the distributed observers with/without communication delay. 

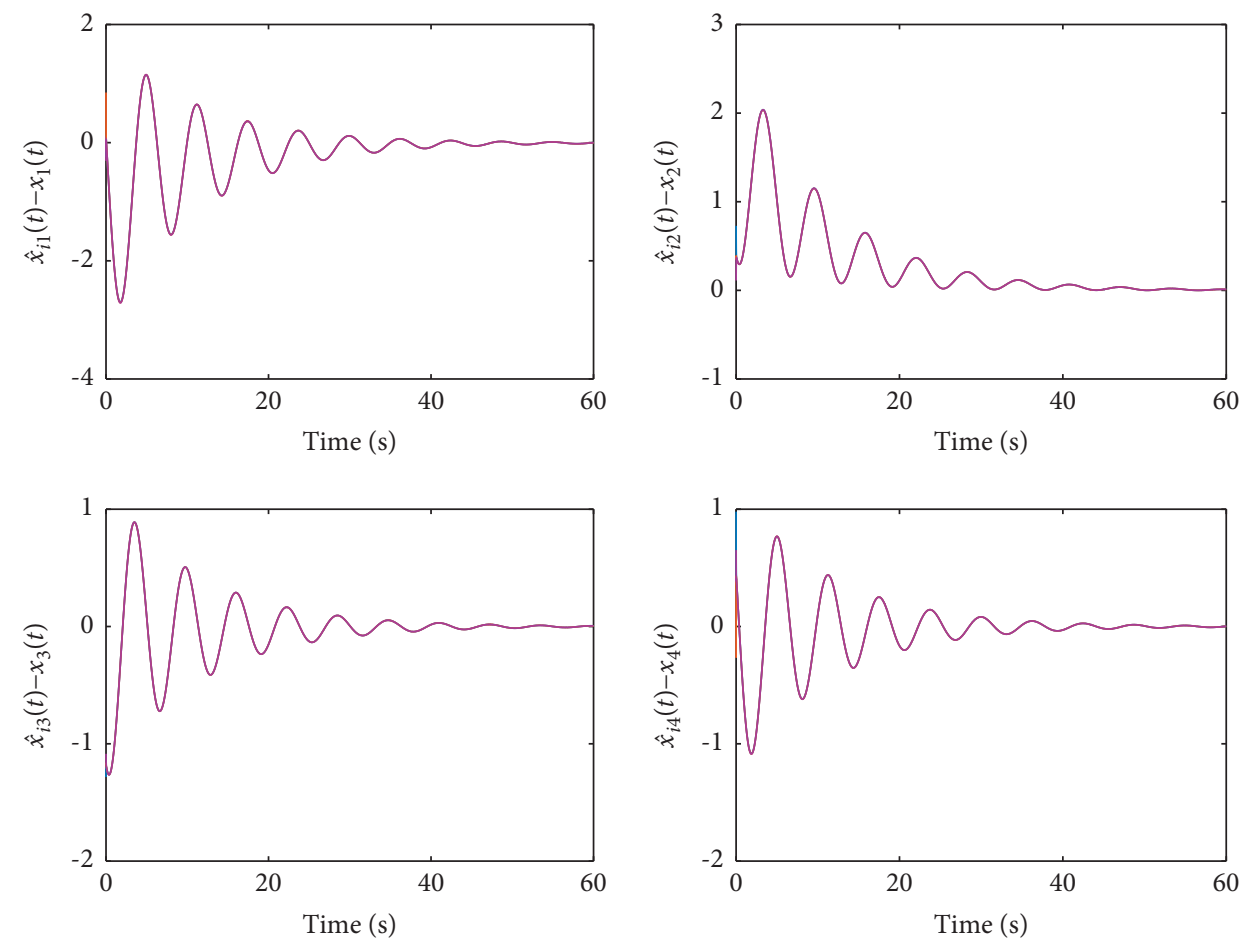

FiguRe 3: Observer errors $\eta_{i}(t)=\widehat{x}_{i}(t)-x(t)(i=1,2,3)$ for time-varying communication delay.
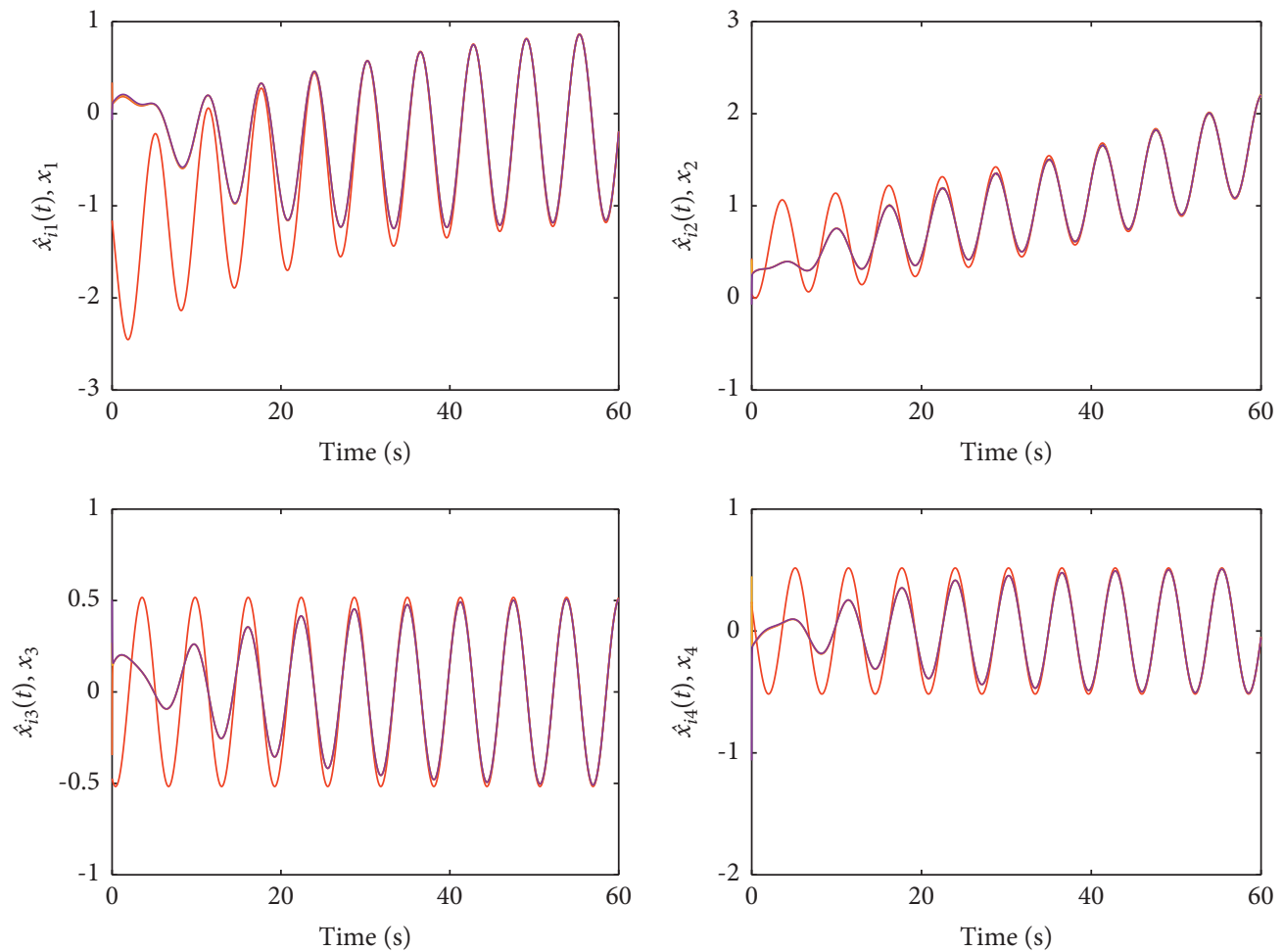

Figure 4: Observer states $\widehat{x}_{i}(t)(i=1,2,3)$ and the plant state $x(t)$ for the delay-free case. 

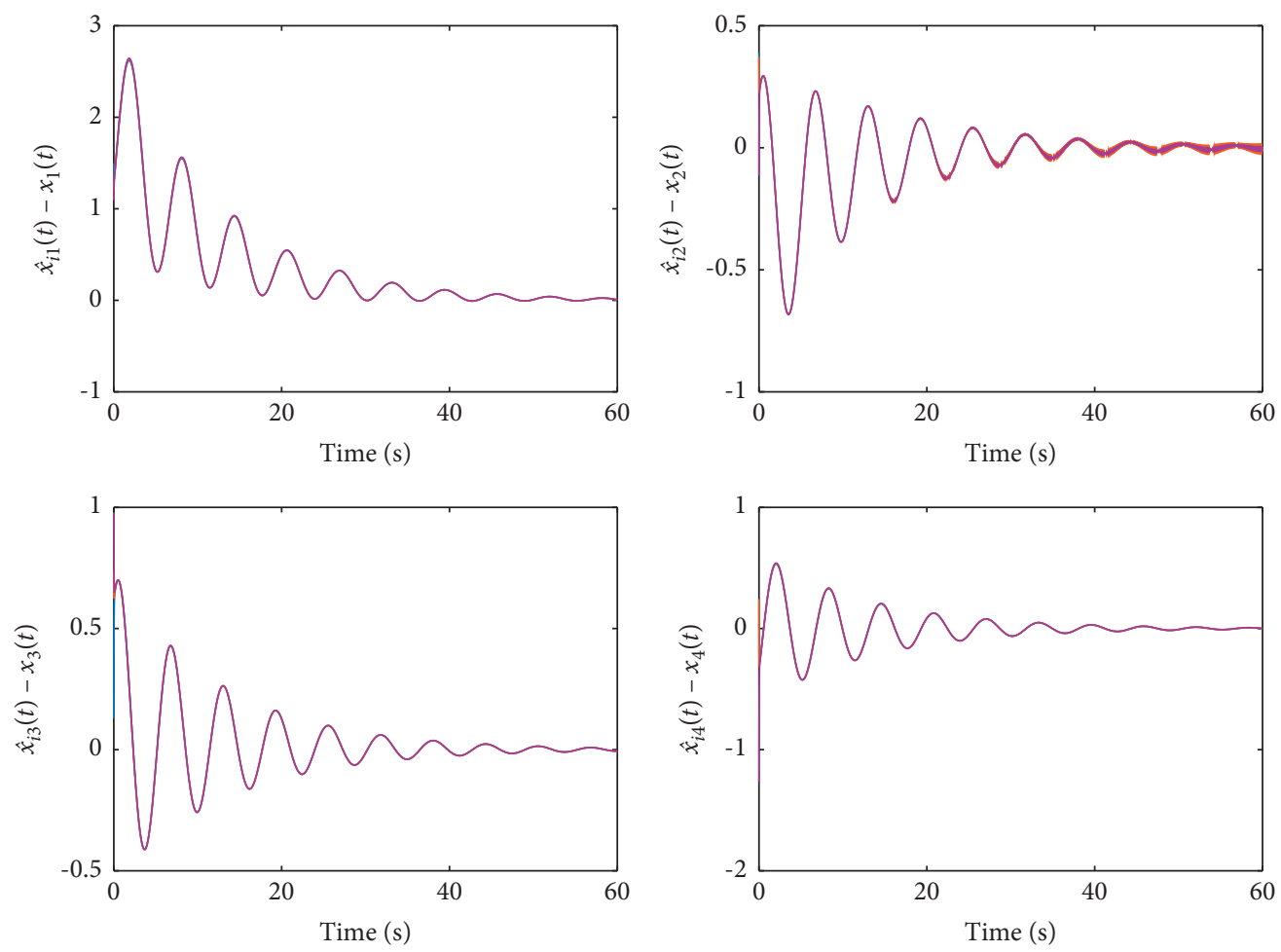

Figure 5: Observer errors $\eta_{i}(t)=\widehat{x}_{i}(t)-x(t)(i=1,2,3)$ for the delay-free case.

\section{Conclusion}

In this paper, we have dealt with the state estimation problem of general continuous-time linear systems, which is solved by the distributed Luenberger observer technique. The structure of the proposed distributed observers comprises networked local observers, in which observers are constructed locally based on its own output measurement and its neighbors according to a given connected communication graph. Remarkably, a network-induced time-varying communication delay has been considered. Two gain parameters, i.e., the gain matrix for local output measurement and the coupling gain for information exchange, have been designed. The local observer gain has been derived by solving a parametric algebraic Riccati equation, while the coupling strength has been determined based on the Lyapunov-Krasovskii stability theorem. Moreover, a bound on the time-varying delay has been also given under which each local observer in the network asymptotically resolves the entire state of the plant.

It should be noted that we focused on linear systems in this paper, and it should be promising to consider some practical issues such as nonlinear plant dynamics, heterogeneous communication delays, transmission errors, and directed communication channels.

\section{Data Availability}

No data were used to support this study.

\section{Conflicts of Interest}

The authors declare that they have no conflicts of interest.

\section{Acknowledgments}

This work was supported in part by the National Natural Science Foundation of China, under Grants 62103115, 11771102, and 61803090, Natural Science Foundation of Guangdong Province, under Grants 2019A1515012109 and 2021A1515011636, Young innovative talents project of general colleges and universities in Guangdong Province, under Grant 2019KQNCX107, Foundation of Key Laboratory of System Control and Information Processing, and Ministry of Education, China.

\section{References}

[1] W. Ren and R. W. Beard, Distributed Consensus in MultiVehicle Cooperative Control, Springer, New York, NY, USA, 2008.

[2] J. Wang, K. Chen, Q. Liu, and Q. Ma, "Observer-based adaptive consensus tracking control for nonlinear multi-agent systems with actuator hysteresis," Nonlinear Dynamics, vol. 95, no. 3, pp. 2181-2195, 2019.

[3] D. Luenberger, "Observers for multivariable systems," IEEE Transactions on Automatic Control, vol. 11, no. 2, pp. 190-197, 1966.

[4] D. Luenberger, "An introduction to observers," IEEE Transactions on Automatic Control, vol. 16, no. 6, pp. 596-602, 1971.

[5] Y. Jin, W. Qi, and G. Zong, "Finite-time synchronization of delayed semi-markov neural networks with dynamic eventtriggered scheme," International Journal of Control, Automation and Systems, vol. 19, no. 6, pp. 2297-2308, 2021.

[6] W. Qi, X. Yang, J. H. Park, J. Cao, and J. Cheng, "Fuzzy smc for quantized nonlinear stochastic switching systems with 
semi-markovian process and application," IEEE Transactions on Cybernetics, pp. 1-10, 2021.

[7] W. Qi, G. Zong, and W. X. Zheng, "Adaptive event-triggered smc for stochastic switching systems with semi-markov process and application to boost converter circuit model," IEEE Transactions on Circuits and Systems I: Regular Papers, vol. 68, no. 2, pp. 786-796, 2020.

[8] J. Wang, H. Zhang, K. Ma, Z. Liu, and C. P. Chen, "Neural adaptive self-triggered control for uncertain nonlinear systems with input hysteresis," IEEE Transactions on Neural Networks and Learning Systems, 2021.

[9] W. Qi, Y. Hou, G. Zong, and C. K. Ahn, "Finite-time event-triggered control for semi-markovian switching cyber-physical systems with fdi attacks and applications," IEEE Transactions on Circuits and Systems I: Regular Papers, vol. 68, no. 6, pp. 2665-2674, 2021.

[10] H.-N. Wu and H.-X. Li, "Robust adaptive neural observer design for a class of nonlinear parabolic pde systems," Journal of Process Control, vol. 21, no. 8, pp. 1172-1182, 2011.

[11] X. Zhang, Y. Han, L. Wu, and Y. Wang, "State estimation for delayed genetic regulatory networks with reaction-diffusion terms," IEEE Transactions on Neural Networks and Learning Systems, vol. 29, no. 2, pp. 299-309, 2018.

[12] A. M. Mohammed, H. Wang, and Y. Tian, "State observer for linear system with unknown input disturbance and sampled and delayed output with measurement noise," Transactions of the Institute of Measurement and Control, vol. 41, no. 3, pp. 749-759, 2019.

[13] R. Mohajerpoor, L. Shanmugam, H. Abdi, S. Nahavandi, and J. H. Park, "Delay-dependent functional observer design for linear systems with unknown time-varying state delays," IEEE Transactions on Cybernetics, vol. 48, no. 7, pp. 2036-2048, 2018.

[14] J. Wang, Y. Huang, T. Wang, C. Zhang, and Y. h. Liu, "Fuzzy finite-time stable compensation control for a building structural vibration system with actuator failures," Applied Soft Computing, vol. 93, Article ID 106372, 2020.

[15] X. Zhang and F. L. Lewis, "Cooperative output regulation of heterogeneous multi-agent systems based on passivity," International Journal of Systems Science, vol. 49, no. 16, pp. 3418-3430, 2018.

[16] L. Xiao, S. Boyd, and S. Lall, "A scheme for robust distributed sensor fusion based on average consensus," in Proceedings of the Fourth International Symposium on Information Processing in Sensor Networks, 2005, pp. 63-70, Boise, ID, USA, April 2005.

[17] R. Olfati-Saber, "Distributed kalman filtering for sensor networks," in Proceedings of the 2007 46th IEEE Conference on Decision and Control, pp. 5492-5498, New Orleans, LA, USA, December 2007.

[18] U. A. Khan and J. M. F. Moura, "Distributing the kalman filter for large-scale systems," IEEE Transactions on Signal Processing, vol. 56, no. 10, pp. 4919-4935, 2008.

[19] M. V. Subbotin and R. S. Smith, "Design of distributed decentralized estimators for formations with fixed and stochastic communication topologies," Automatica, vol. 45, no. 11, pp. 2491-2501, 2009.

[20] D. Viegas, P. Batista, P. Oliveira, and C. Silvestre, "Decentralized observers for position and velocity estimation in vehicle formations with fixed topologies," Systems \& Control Letters, vol. 61, no. 3, pp. 443-453, 2012.

[21] W. Zhou, J. Fu, H. Yan, X. Du, Y. Wang, and H. Zhou, "Eventtriggered approximate optimal path-following control for unmanned surface vehicles with state constraints," IEEE
Transactions on Neural Networks and Learning Systems, pp. 1-15, 2021.

[22] V. Delouille, R. Neelamani, and R. Baraniuk, "Robust distributed estimation in sensor networks using the embedded polygons algorithm," in Proceedings of the Third International Symposium on Information Processing in Sensor Networks, 2004, pp. 405-413, Berkeley, CA, USA, April 2004.

[23] R. Olfati-Saber, "Distributed kalman filter with embedded consensus filters," in Proceedings of the 44th IEEE Conference on Decision and Control, pp. 8179-8184, Seville, Spain, December 2005.

[24] S. Park and N. C. Martins, "An augmented observer for the distributed estimation problem for LTI systems," in Proceedings of the 2012 American Control Conference (ACC), pp. 775-6780, Montreal, QC, Canada, June 2012.

[25] S. Park and N. C. Martins, "Design of distributed LTI observers for state omniscience," IEEE Transactions on Automatic Control, vol. 62, no. 2, pp. 561-576, 2017.

[26] H. Zhu, K. Liu, J. Lü, Z. Lin, and Y. Chen, "On the cooperative observability of a continuous-time linear system on an undirected network," in Proceedings of the 2014 International Joint Conference on Neural Networks (IJCNN), pp. 2940-2944, Beijing, China, July 2014.

[27] K. Liu, H. Zhu, and J. Lü, "Cooperative stabilization of a class of LTI plants with distributed observers," IEEE Transactions on Circuits and Systems I: Regular Papers, vol. 64, no. 7, pp. 1891-1902, 2017.

[28] K. Liu, Y. Chen, Z. Duan, and J. Lü, “Cooperative output regulation of LTI plant via distributed observers with local measurement," IEEE Transactions on Cybernetics, vol. 48, no. 7, pp. 2181-2191, 2018.

[29] K. Chen, J. Wang, Z. Zhao, G. Lai, and Y. Lyu, "Output consensus of heterogeneous multiagent systems: a distributed observer-based approach," IEEE Transactions on Systems, Man, and Cybernetics: Systems, pp. 1-7, 2020.

[30] T. Kim, H. Shim, and D. D. Cho, "Distributed luenberger observer design," in Proceedings of the 2016 IEEE 55th Conference on Decision and Control (CDC), pp. 6928-6933, Las Vegas, NV, USA, December 2016.

[31] W. Han, H. L. Trentelman, Z. Wang, and Y. Shen, "A simple approach to distributed observer design for linear systems," IEEE Transactions on Automatic Control, vol. 64, no. 1, pp. 329-336, 2019.

[32] Z. Zhiwei Gao and S. X. Ding, "State and disturbance estimator for time-delay systems with application to fault estimation and signal compensation," IEEE Transactions on Signal Processing, vol. 55, no. 12, pp. 5541-5551, 2007.

[33] Z. Zhiwei Gao, T. Breikin, and H. Hong Wang, "Reliable observer-based control against sensor failures for systems with time delays in both state and input," IEEE Transactions on Systems, Man, and Cybernetics-Part A: Systems and Humans, vol. 38, no. 5, pp. 1018-1029, 2008.

[34] J. R. Moyne and D. M. Tilbury, "The emergence of industrial control networks for manufacturing control, diagnostics, and safety data," Proceedings of the IEEE, vol. 95, no. 1, pp. 29-47, 2007.

[35] F. Liu, H. Gao, J. Qiu, S. Yin, J. Fan, and T. Chai, "Networked multirate output feedback control for setpoints compensation and its application to rougher flotation process," IEEE Transactions on Industrial Electronics, vol. 61, no. 1, pp. 460-468, 2014.

[36] H. J. Savino, C. R. P. dos Santos, F. O. Souza, L. C. A. Pimenta, M. de Oliveira, and R. M. Palhares, "Conditions for consensus of multi-agent systems with time-delays and uncertain 
switching topology," IEEE Transactions on Industrial Electronics, vol. 63, no. 2, pp. 1258-1267, 2016.

[37] K. Chen, J. Wang, Y. Zhang, and Z. Liu, "Leader-following consensus for a class of nonlinear strick-feedback multiagent systems with state time-delays," IEEE Transactions on Systems, Man, and Cybernetics: Systems, vol. 50, no. 7, pp. 2351-2361, 2020.

[38] Z. Gao, "Estimation and compensation for lipschitz nonlinear discrete-time systems subjected to unknown measurement delays," IEEE Transactions on Industrial Electronics, vol. 62, no. 9, pp. 5950-5961, 2015.

[39] K. Liu, J. Lü, and Z. Lin, "Design of distributed observers in the presence of arbitrarily large communication delays," IEEE Transactions on Neural Networks and Learning Systems, vol. 29, no. 9, pp. 4447-4461, 2018.

[40] H. Basu and S. Y. Yoon, "Distributed state estimation by a network of observers under communication and measurement delays," Systems \& Control Letters, vol. 133, 2019.

[41] C. Godsil and G. F. Royle, Algebraic Graph Theory, Springer Science \& Business Media, Berlin, Germany, 2001.

[42] B. Bin Zhou, Z. Zongli Lin, and G.-R. Guang-Ren Duan, "Properties of the parametric Lyapunov equation-based lowgain design with applications in stabilization of time-delay systems," IEEE Transactions on Automatic Control, vol. 54, no. 7, pp. 1698-1704, 2009.

[43] S. Boyd, L. El Ghaoui, E. Feron, and V. Balakrishnan, Linear Matrix Inequalities in System and Control Theory, SIAM, Philadelphia, PA, USA, 1994.

[44] K. Gu, J. Chen, and V. L. Kharitonov, Stability of Time-Delay Systems, Springer Science \& Business Media, Berlin, Germany, 2003.

[45] E. Fridman, Introduction to Time-Delay Systems: Analysis and Control, Springer, Berlin, Germany, 2014.

[46] Z. Li, W. Ren, X. Liu, and M. Fu, "Consensus of multi-agent systems with general linear and Lipschitz nonlinear dynamics using distributed adaptive protocols," IEEE Transactions on Automatic Control, vol. 58, no. 7, pp. 1786-1791, 2013.

[47] W. Yu, W. Ren, W. X. Zheng, G. Chen, and J. Lü, "Distributed control gains design for consensus in multi-agent systems with second-order nonlinear dynamics," Automatica, vol. 49, no. 7, pp. 2107-2115, 2013. 